\title{
Self-assembly and hydrogel formation ability of Fmoc-dipeptides comprising a-methyl-L-phenylalanine
}

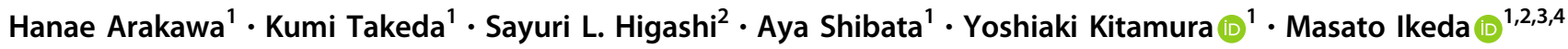

Received: 14 November 2019 / Revised: 8 December 2019 / Accepted: 8 December 2019 / Published online: 10 January 2020

(c) The Author(s) 2020. This article is published with open access

\begin{abstract}
Various biofunctional hydrogel materials can be fabricated in aqueous media through the self-assembly of peptide derivatives, forming supramolecular nanostructures and their three-dimensional networks. In this study, we describe the self-assembly of new Fmoc-dipeptides comprising $\alpha$-methyl-L-phenylalanine. We found that the position and number of methyl groups introduced onto the $\alpha$ carbons of the Fmoc-dipeptides by $\alpha$-methyl-L-phenylalanine have a marked influence on the morphology of the supramolecular nanostructure as well as the hydrogel (network) formation ability.
\end{abstract}

\section{Introduction}

Supramolecular nanostructures, which consist of selfassembling peptide derivatives, have attracted rapidly increasing attention for the fabrication of various biofunctional materials [1-9]. Recent seminal works have revealed that short peptides bearing an aromatic group at their $\mathrm{N}$-termini have a robust potential to self-assemble into supramolecular nanostructures under aqueous conditions with subsequent hydrogel formation, facilitating active exploration [10-17]. Rediscovery that simple dipeptides bearing the Fmoc

These authors contributed equally: Hanae Arakawa, Kumi Takeda

Supplementary information The online version of this article (https:// doi.org/10.1038/s41428-019-0301-5) contains supplementary material, which is available to authorized users.

Masato Ikeda

m_ikeda@gifu-u.ac.jp

1 Department of Life Science and Chemistry, Graduate School of Natural Science and Technology, Gifu University, 1-1 Yanagido, Gifu 501-1193, Japan

2 United Graduate School of Drug Discovery and Medical Information Sciences, Gifu University, 1-1 Yanagido, Gifu 5011193, Japan

3 Center for Highly Advanced Integration of Nano and Life Sciences (G-CHAIN), Gifu University, 1-1 Yanagido, Gifu 5011193, Japan

4 Institute of Nano-Life-Systems, Institute of Innovation for Future Society, Nagoya University, Furu-cho, Chikusa-ku, Nagoya 4648603, Japan (9-fluorenylmethyloxycarbonyl) group (one of the most typical protecting groups) give rise to hydrogels, as reported by $\mathrm{Xu}$ and coworkers [10], has facilitated the research activity [4]. In addition, among such self-assembling peptides, an aromatic phenylalanine-phenylalanine dipeptide $[\mathbf{F F},(\mathbf{F}$ : L-phenylalanine)] is one of the simplest and most advantageous scaffolds used to fabricate a variety of supramolecular nanostructures $[18,19]$. In fact, two research groups simultaneously reported that the FF dipeptide bearing the Fmoc group (Fmoc-FF) could form stable supramolecular hydrogels due to the formation of a supramolecular nanofiber network, which were utilized as scaffold materials for cell cultures $[11,12]$.

As discrete structural derivatives of natural peptides can be synthesized, $\alpha, \alpha$-disubstituted $\alpha$-amino acids ( $\alpha \alpha \mathrm{AAs}$ ) can offer a distinctive opportunity to modulate the morphology of supramolecular nanostructures [20, 21]. It is well recognized that peptides consisting mainly of $\alpha$-aminoisobutyric acid (Aib), which is one of the most actively investigated $\alpha \alpha$ AAs, adopt a $3_{10}$-helix instead of an $\alpha$-helix $[21,22]$. Control of the helicity of the $3_{10}$-helix in response to various chiral stimuli has been actively investigated [22]. On the other hand, the self-assembly of peptides containing $\alpha \alpha \mathrm{AAs}$ has remained unexplored. Hammer and coworkers demonstrated that a peptide containing $\alpha \alpha$ AAs inhibits $\beta$-amyloid formation [23]. Moreover, Moretto and coworkers developed unique peptides containing $\alpha \alpha \mathrm{AA}$, which have two azobenzene moieties as side chains that are capable of participating in the photoresponsive transformations of supramolecular nanostructures [24]. Due to limited examples $[25,26]$, the systematic construction of peptides 
containing $\alpha \alpha \mathrm{AAs}$ and the investigation of their selfassembly are significant in understanding the influence of subtle but precise chemical modifications of constituent peptides on the ability of these peptides to form supramolecular nanostructures.

Herein, we describe the self-assembly and supramolecular hydrogel formation ability of Fmoc-dipeptides (Fmoc-XXs, Fmoc = 9-fluorenylmethyloxycarbonyl, $\mathbf{X}=$ $\mathbf{F}$ or ${ }^{\mathbf{D}} \mathbf{F}$ or $\mathbf{m F}$ ) comprising $\alpha$-methyl-L-phenylalanine $(\mathbf{m F})$ as one of the $\alpha \alpha \mathrm{AAs}$ and/or L-phenylalanine $(\mathbf{F})$ or D-phenylalanine $\left({ }^{\mathbf{D}} \mathbf{F}\right)$. To our knowledge, the influence of such chemical modification of the Fmoc-FF at the $\alpha$ carbons on the self-assembling propensity is unexplored. Due to a reliable and scalable synthetic procedure with asymmetric phase-transfer catalysts, developed by Maruoka and coworkers, a variety of $\alpha \alpha \mathrm{AAs}$, including $\mathbf{~ m F}$, with high purity are now commercially available [27, 28]. In this study, we found that the position and number of methyl groups introduced onto the $\alpha$ carbons of the Fmocdipeptides (Fig. 1) have an unexpectedly marked influence on the morphology of the supramolecular nanostructure as well as the hydrogel formation ability.

\section{Materials and methods}

\section{Experimental generals}

Unless stated otherwise, all commercial reagents were used as received. All water used in the experiments was ultra-pure water obtained from a Millipore system with a specific resistance of $18 \mathrm{M} \Omega \cdot \mathrm{cm}$. Thin-layer chromatography was performed on silica gel $60 \mathrm{~F}_{254}$ (Merck). ${ }^{1} \mathrm{H}$ and ${ }^{13} \mathrm{C}$ NMR spectra were obtained on a JEOL JNM ECS400 spectrometer $\left(400 \mathrm{MHz}\right.$ for ${ }^{1} \mathrm{H}, 100 \mathrm{MHz}$ for ${ }^{13} \mathrm{C}$ ) with tetramethylsilane (TMS) or residual nondeuterated solvents as the internal references. Multiplicities are abbreviated as follows: $\mathrm{s}=$ singlet, $\mathrm{d}=$ doublet, $\mathrm{t}=$ triplet, $\mathrm{q}=$ quartet, $\mathrm{m}=$ multiplet, $\mathrm{dd}=$ double doublet, and $\mathrm{br}=$ broad. MALDI-TOF mass spectra were recorded using a Shimadzu AXIMA-CFR plus mass spectrometer. High-resolution mass spectra were obtained on a JEOL JMS-T100LP AccuTOF LC-plus (ESI) or a Waters Xevo QTof (ESI) mass spectrometer. Fluorescence spectra were obtained using a PerkinElmer LS-55 fluorescence spectrometer. a<smiles>O=C(N[C@@H](Cc1ccccc1)C(=O)N[C@@H](Cc1ccccc1)C(=O)O)OCC1c2ccccc2-c2ccccc21</smiles>

Fmoc-FF<smiles>O=C(N[C@@H](Cc1ccccc1)C(=O)NC(Cc1ccccc1)C(=O)O)OCC1c2ccccc2-c2ccccc21</smiles>

Fmoc-FmF

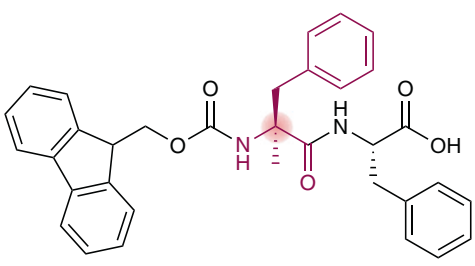

Fmoc-mFF<smiles>CC(NC(=O)[C@H](Cc1ccccc1)NC(=O)OCC1c2ccccc2-c2ccccc21)C(=O)O</smiles>

Fmoc-mFmF<smiles>O=C(NC(Cc1ccccc1)C(=O)NC(Cc1ccccc1)C(=O)O)OCC1c2ccccc2-c2ccccc21</smiles>

Fmoc- ${ }^{D_{F m F}}$

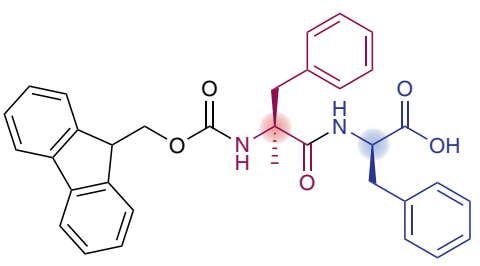

Fmoc-mF $F^{\mathrm{D}}$ b

\begin{tabular}{|c|c|c|}
\hline & 0.20 & $0.40 / w t \%$ \\
\hline Fmoc-FF & Gel & Gel \\
\hline Fmoc-mFmF & tSol & tSol \\
\hline Fmoc-FmF & tSol & tSol \\
\hline Fmoc-mFF & pGel & Gel \\
\hline Fmoc-DFmF & pGel & Gel \\
\hline Fmoc-mFDF & tSol & tSol \\
\hline
\end{tabular}

Fig. 1 Hydrogel formation ability of Fmoc-XXs. a Chemical structures of Fmoc-dipeptides (Fmoc-XXs, $\mathbf{X}=\mathbf{F}$ or ${ }^{\mathbf{D}} \mathbf{F}$ or $\mathbf{m F}$ ) comprising phenylalanine and $\alpha$-methyl-phenylalanine. $\mathbf{b}$ Summary of the gelation tests (Gel transparent or translucent gel, $p G e l$ partial gel, $t$ Sol turbid solution, Sol clear solution) of Fmoc-XXs. Conditions: $100 \mathrm{mM}$
HEPES-NaOH (pH 7.4) containing DMSO (2.0 vol\% for $0.20 \mathrm{wt} \%$ of Fmoc-XXs and $4.0 \mathrm{vol} \%$ for $0.40 \mathrm{wt} \%$ of Fmoc-XXs). Fmoc 9fluorenylmethyloxycarbonyl, $\boldsymbol{F}$ L-phenylalanine, ${ }^{D} \boldsymbol{F}$ D-phenylalanine, $\boldsymbol{m} \boldsymbol{F} \alpha$-methyl-L-phenylalanine 


\section{Solid-phase synthesis of Fmoc-dipeptides (Fmoc-XXs)}

Syntheses of Fmoc-dipeptides (Fmoc-XXs) were carried out in the solid phase by using a multiple reaction device (HiPep Laboratories, PetiSyzer) according to conventional solid-phase peptide synthesis protocols. All reactions were carried out in cartridges with polypropylene frits (HiPep Laboratories, LibraTube) using 2-chlorotrityl chloride resin (Watanabe, 100-200 mesh, 1\% DVB, 1.6 mmol/g). The condensation reaction was carried out in the presence of Fmoc-amino acid (3.0 eq.), 1-[bis(dimethylamino) methyliumyl]-1H-benzotriazole-3-oxide hexafluorophosphate (HBTU, 3.0 eq.), 1-hydroxybenzotriazole hydrate (HOBT $\cdot \mathrm{H}_{2} \mathrm{O}, 3.0$ eq.), and $N, N$-diisopropylethylamine (DIEA, 6.0 eq.) in $N$-methylpyrrolidone at room temperature (from 1 to $3 \mathrm{~h}$ ). For the condensation reactions with Fmoc-mF, 1-[bis(dimethylamino)methyliumyl]-1 $H$ 1,2,3-triazolo[4,5-b]pyridine-3-oxide hexafluorophosphate (HATU, 3.0 eq.) was utilized instead of HBTU [29]. Fmoc deprotection was achieved with $20 \%$ piperidine in $N$, $\mathrm{N}$-dimethylformamide (from 5 to $15 \mathrm{~min}$ ). After the final step, a mixture of hexafluoroisopropanol (HFIP)-dichloromethane $(1: 4 v / v)$ was added, and the resin was removed by filtration and washed with the cleavage mixture. The collected filtrate was concentrated under reduced pressure, and the peptide was precipitated by the addition of diethyl ether and hexane. Each obtained peptide was analyzed by ${ }^{1} \mathrm{H}$ and ${ }^{13} \mathrm{C}$ NMR and ESI-TOF-HRMS (Electronic supplementary materials).

\section{Preparation of hydrogel or dispersion}

Gelation ability was evaluated by an inverted tube test [30]. Typically, Fmoc-XX powder was dissolved in dimethyl sulfoxide (DMSO), and the resultant stock solution (10 wt $\%, 4.0 \mu \mathrm{L})$ was mixed with aqueous buffer [100 $\mathrm{mM} 4-(2-$ hydroxyethyl)-1-piperazineethanesulfonic acid (HEPES) (pH 7.4), $196 \mu \mathrm{L}$ ] inside a glass vial (inner diameter: 9.0 $\mathrm{mm})$. The suspension was heated until a homogeneous solution was obtained. The solution was left to cool at room temperature without any disturbance. Gel formation was determined by whether the sample flows or not when the glass vial is inverted.

\section{Transmission electron microscope (TEM) observation}

A sample $(\sim 10 \mu \mathrm{L})$ was dropped on a copper TEM grid covered by an elastic carbon-support film $(20-25 \mathrm{~nm})$ with filter paper underneath, and the excess solution was immediately blotted with filter paper. The TEM grid was dried under a reduced pressure for at least $6 \mathrm{~h}$ prior to TEM observation. TEM images were acquired using a Hitachi
$\mathrm{H}-7000$ (accelerating voltage: $100 \mathrm{kV}$ ) equipped with a CCD camera and AMTV600 software.

\section{Dynamic light scattering (DLS) measurement}

DLS measurements were performed on a Malvern Zetasizer Nano equipped with a He-Ne laser $(633 \mathrm{~nm})$. Aqueous solutions were filtered using a Millipore membrane filter $(0.45 \mu \mathrm{m}$ pore size $)$ before the measurements. The particle size distribution was derived from a deconvolution of the measured intensity autocorrelation function by the "general purpose mode" (nonnegative linear least-squares) algorithm included in the software of the instrument.

\section{Confocal laser scanning microscope (CLSM) observation}

A freshly prepared homogeneous solution $(0.20$ or $0.40 \mathrm{wt}$ $\%, 100 \mathrm{mM}$ HEPES (pH 7.4), $20 \mu \mathrm{L}$ ) of Fmoc-XXs obtained by heating in a glass vial was transferred to a microtube and left to cool at room temperature without any disturbance and was subsequently mixed with a DMSO solution of Nile red $(0.3 \mathrm{mM}$ (final concentration is $14 \mu \mathrm{M}$ ), $1.0 \mu \mathrm{L})$. The gel $(\sim 20 \mu \mathrm{L})$ was spotted on a glass coverslip (diameter: $25 \mathrm{~mm}$, thickness: $0.13-0.17 \mathrm{~mm}$, Fisher Scientific) and placed in an Attofluor cell chamber (Thermo Fisher Scientific) with water drops $(50 \mu \mathrm{L})$ around the sample drop to avoid dryness. The sample observed using an inverted CLSM (FV1000-D, Olympus) equipped with an Ar laser $(488 \mathrm{~nm})$ and LED laser $(559 \mathrm{~nm})$. A $60 \times$ (numerical aperture $(\mathrm{NA})=1.49$ ) oil objective was employed to obtain images. The obtained images analyzed by the acquisition software FV10-ASW4.2 supplied with a microscope. The images were also quantified and analyzed by Fiji (freely available at https://fiji.sc/) [31, 32].

\section{Critical aggregation concentration (CAC) determinations}

CACs of Fmoc-XXs were evaluated by a thioflavin T (ThT) assay [33]. The solutions of Fmoc-XXs [100 mM HEPES$\mathrm{NaOH}$ (pH 7.4) containing DMSO (less than 2.0 vol\%)] at various concentrations containing ThT $(15 \mu \mathrm{M})$ were prepared. Fluorescence spectra $\left(\lambda_{\mathrm{ex}}=440 \mathrm{~nm}\right)$ of the solutions were obtained using a PerkinElmer LS-55 fluorescence spectrometer with a quartz cell $(1.0 \mathrm{~cm} \times 0.2 \mathrm{~cm})$. Fluorescence intensities at $\lambda_{\mathrm{em}}=494 \mathrm{~nm}$ were plotted against the concentration of Fmoc-XXs to evaluate the CACs.

\section{Rheological measurement}

Dynamic frequency and strain sweep experiments were carried out on a TA Instruments AR-G2 rheometer using a 
Fig. 2 CAC determinations of Fmoc-XXs. a Plots of fluorescence intensity of ThT at $494 \mathrm{~nm}$ as a function of Fmocdipeptide (Fmoc-XXs, $\mathbf{X}=\mathbf{F}$ or ${ }^{D} \mathbf{F}$ or $\mathbf{m F}$ ) concentrations. b CACs $(\mu \mathrm{M})$ estimated from plots a. Conditions: $[\mathrm{ThT}]=15 \mu \mathrm{M}$, $100 \mathrm{mM}$ HEPES-NaOH $(\mathrm{pH}$ 7.4) containing DMSO (2.0 vol $\%), 25{ }^{\circ} \mathrm{C}$

Fig. 3 TEM observations of selfassembled nanostructures of Fmoc-XXs. Representative TEM images of (a) Fmoc-FF, (b) Fmoc-mFmF, (c) FmocFmF, (d) Fmoc- ${ }^{{ }^{2}} \mathbf{F m F}$, (e) Fmoc-mFF, and (f) Fmoc$\mathbf{m} \mathbf{F}^{\mathbf{D}} \mathbf{F}$ and (iii) histogram analysis of the diameter distributions of nanospheres (c) and beads (d). Insets in the TEM images show hydrogels or dispersions in side-tilting glass vials $(0.20 \mathrm{wt} \%)$. Scale bar: 500 nm (except a $1 \mu \mathrm{m})$

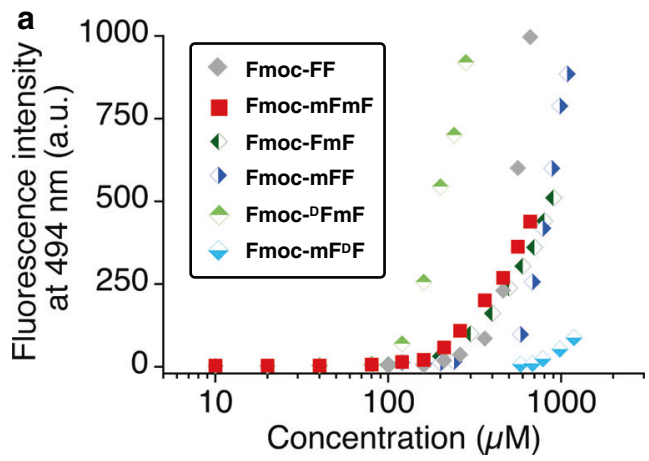

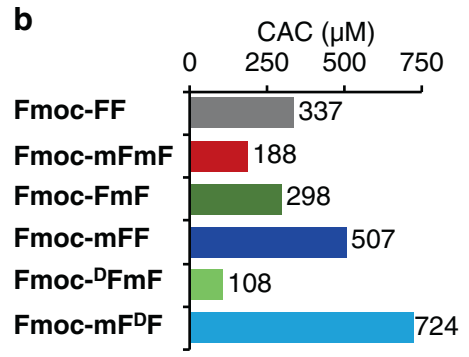
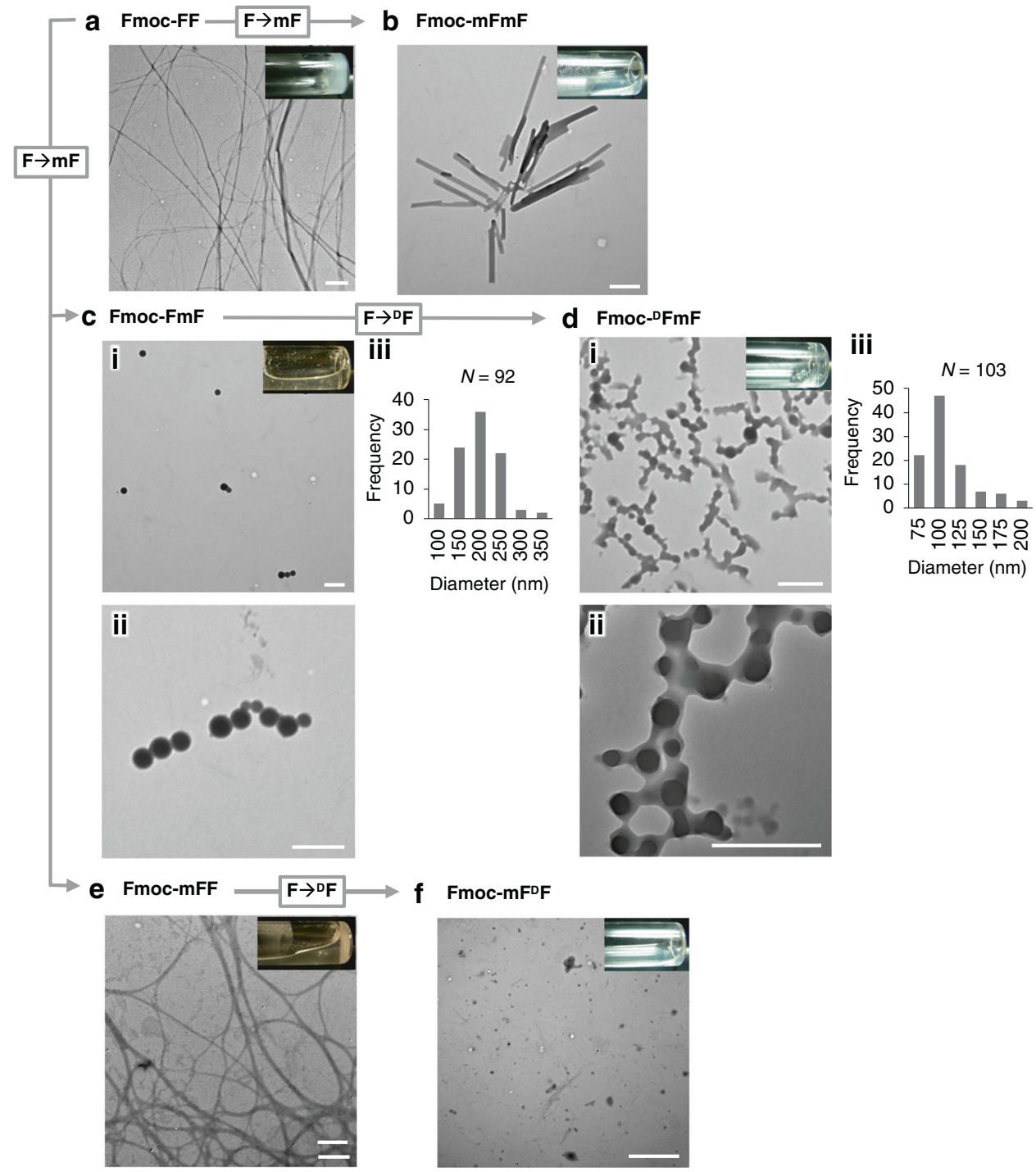

20-mm stainless steel parallel plate (the temperature of the plate was controlled at $25^{\circ} \mathrm{C}$ by a Peltier system) at a gap of $1.0 \mathrm{~mm}$. Hydrogel samples were placed on the plate. All the gels showed an almost linear viscoelastic regime up to $1.0 \%$ strain (frequency: $1.0 \mathrm{rad} / \mathrm{s}$ ). Therefore, a frequency sweep $(0.1-100 \mathrm{rad} / \mathrm{s})$ was performed under $0.2 \%$ strain.

\section{Results and discussion}

Five distinct Fmoc-dipeptides, Fmoc-mFmF, Fmoc-FmF, Fmoc-mFF, Fmoc- ${ }^{\mathbf{D}}$ FmF, and Fmoc-mF ${ }^{\mathbf{D}} \mathbf{F}$ (Fig. 1a), were newly synthesized following standard Fmoc-based solidphase peptide synthesis using a 2-chlorotrityl chloride resin. 


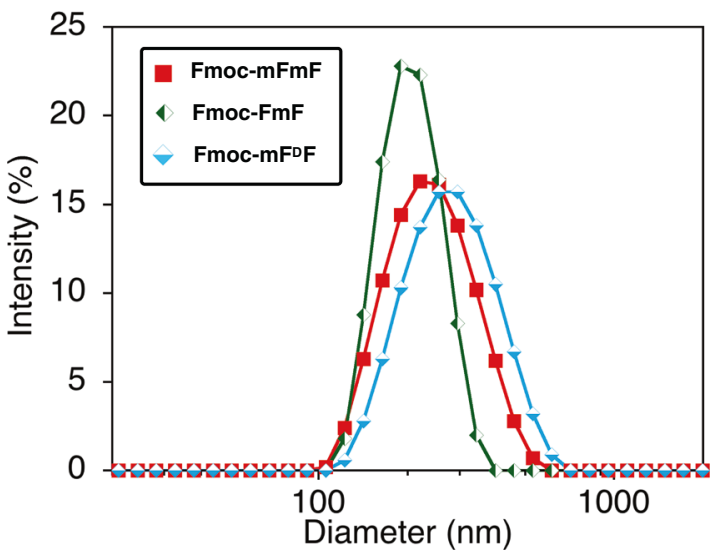

Fig. 4 Size distributions estimated by DLS measurements for selfassembled nanostructures of Fmoc-mFmF, Fmoc-FmF, and Fmoc$\mathbf{m F}^{\mathbf{D}} \mathbf{F}$. Conditions: $[\mathbf{F m o c}-\mathbf{X X s}]=0.20 \mathrm{wt} \%, 100 \mathrm{mM}$ HEPES-NaOH (pH 7.4) containing DMSO (2.0 vol\%), $25^{\circ} \mathrm{C}$

Note that, since coupling with Fmoc-mF was slow and inefficient, most likely due to the steric hindrance of the $\alpha$ methyl group, the amount of Fmoc-mF used was increased, and HATU was used as the coupling reagent instead of HBTU. All Fmoc-XXs showed reasonable solubility toward DMSO and HFIP. A conventional tube-inversion method [30] was applied to evaluate the hydrogel formation ability. As summarized in Fig. 1b, Fmoc-FF, which is employed as a typical hydrogelator for control experiments, produced hydrogels at concentrations above $0.20 \mathrm{wt} \%$. Gratifyingly, we found that both Fmoc-mFF and Fmoc${ }^{\mathbf{D}} \mathbf{F m F}$ showed the ability to form supramolecular hydrogels at $0.20 \mathrm{wt} \%$, suggesting the formation of networked supramolecular nanostructures. In contrast, Fmoc-mFmF, Fmoc-FmF, and Fmoc-mF $\mathbf{F}^{\mathbf{D}} \mathbf{F}$ showed no hydrogel formation ability; instead, rather stable suspensions were obtained, suggesting the formation of nonnetworked but self-assembled nanostructures. These differences in hydrogel formation ability, which can be attributed to the introduction of the $\alpha$-methyl group and/or the chirality of the phenylalanine group in the Fmoc-FF dipeptide scaffold, were further investigated in the following study.

To compare the self-assembling properties of Fmoc$\mathbf{X X}$ s, we investigated their CACs by using a widely used environment-responsive and preferentially $\beta$-sheet binding fluorescence dye, thioflavin $\mathrm{T}$ (ThT) [33]. Fig. 2b summarizes the CACs evaluated from the concentrationdependent fluorescence intensity plot (Fig. 2a). Here, Fmoc- ${ }^{\mathbf{D}} \mathbf{F m} \mathbf{F}$ showed the lowest CAC and the strongest propensity to self-assemble, while $\mathbf{F m o c}-\mathbf{m} \mathbf{F}^{\mathbf{D}} \mathbf{F}$ showed the highest CAC and a significantly lower fluorescence intensity, suggesting its weaker propensity to self-assemble and adopt $\beta$-sheet structures. Additionally, this result indicates that the methyl groups introduced onto the $\alpha$ carbons have a significant influence on the self-assembling propensity and suggests that there would be a tendency for the methyl group at the $\mathrm{N}$-terminus to enhance selfassembly and a tendency for that at the C-terminus to attenuate it.

TEM observations were carried out to gain insight into the morphology of the supramolecular nanostructures of Fmoc-XXs. Consistent with previous reports [10, 11], the network structures of the nanofibers were clearly visualized for Fmoc-FF (Fig. 3a). On the other hand, nonnetworked nanorod structures were found for Fmoc-mFmF, a derivative containing two $\alpha$-methyl groups (Fig. $3 b$ ). When one F of Fmoc-FF was replaced with $\mathbf{m F}$, highly spherical nanostructures were observed for Fmoc-FmF, as shown in Fig. 3c-i, ii. The average diameter was $174 \pm 50 \mathrm{~nm}$ (the histogram analysis is shown in Fig. 3c-iii). In good agreement with the size estimated from TEM images, DLS experiments showed an intensity distribution with a peak corresponding to an average hydrodynamic diameter of $209 \pm 48 \mathrm{~nm}$ with a relatively narrow distribution (Fig. 4 and Table 1). In sharp contrast, the network structures of fibrous architectures with diameters of several tens of $\mathrm{nm}$ were observed for Fmoc-mFF (Fig. 3e), which should be responsible for the formation of a supramolecular hydrogel. Clearly, the position and the number of methyl groups introduced onto the $\alpha$ carbons of the original Fmoc-FF have a profound influence on the morphology of supramolecular nanostructures and the hydrogel formation ability.

We also surveyed the influence of the chirality of phenylalanine on the morphology of supramolecular nanostructures [34-36] for both Fmoc-mFF and FmocFmF. Marchesan and coworkers systematically investigated the supramolecular hydrogel formation ability of peptides that contain D-amino acid residues [34, 35]. For example, although the tripeptides $\mathbf{V F F}$ (V, valine) and FFV failed to self-assemble at physiological $\mathrm{pH},{ }^{\mathbf{D}} \mathbf{V F F}$ $\left({ }^{\mathbf{D}} \mathbf{V}\right.$, D-valine) and ${ }^{\mathbf{D}} \mathbf{F F V}$ successfully self-assembled to form hydrogels [35]. In addition, Banerjee and coworkers reported that the incorporation of D-amino acid into BocFFF (Boc, tert-butoxycarbonyl) enables modulation of the physiological stability as well as the stiffness of the hydrogels [36]. Herein, we found that $\mathbf{F m o c}-\mathbf{m} \mathbf{F}^{\mathbf{D}} \mathbf{F}$, as a result of chirality inversion at the C-terminus of FmocmFF, does not show well-defined nanostructures (Fig. 3f). In contrast, clusters of spherical nanostructures were observed for Fmoc- ${ }^{\mathbf{D}} \mathbf{F m F}$ (Fig. 3d-i, ii). The average diameter of the spherical nanostructures was $96 \pm 31 \mathrm{~nm}$ (the histogram analysis is shown in Fig. 3d-iii). This result suggests (colloidal) hydrogel formation [37], which has rarely been reported for short self-assembling peptides [38, 39].

To further investigate the self-assembled morphology under wet conditions and to gain insight into the mechanism 
Table 1 Summary of selfassembled morphology and hydrogel formation ability of Fmoc-XXs and DLS results

Fig. 5 CLSM observations of hydrogels. Representative CLSM images of (a) Fmoc-FF, (b) Fmoc-mFF, and (c) Fmoc${ }^{D}$ FmF. Scale bar: $30 \mu \mathrm{m}$. Conditions: $[\mathbf{F m o c}-\mathbf{X X s}]=$ $0.20 \mathrm{wt} \% 100 \mathrm{mM}$ HEPES$\mathrm{NaOH}(\mathrm{pH} 7.4)$ containing DMSO $(2.0$ vol $\%)$, [Nile red $]=$ $14 \mu \mathrm{M}, 25^{\circ} \mathrm{C}$

\begin{tabular}{|c|c|c|c|}
\hline Fmoc-XXs & Morphology ${ }^{a}$ & Hydrogel formation below $0.40 \mathrm{wt} \%^{\mathrm{b}}$ & Size $(\mathrm{nm})$ estimated by DLS \\
\hline Fmoc-FF & Fiber (networked) & $\checkmark$ & \\
\hline Fmoc-mFmF & Rod & & $250 \pm 83$ \\
\hline Fmoc-FmF & Sphere & & $209 \pm 48$ \\
\hline Fmoc-mFF & Fiber (networked) & $\checkmark$ & \\
\hline Fmoc- ${ }^{D} \mathbf{F m F}$ & Sphere (clustered) & $\checkmark$ & \\
\hline Fmoc-mF ${ }^{\mathrm{D}} \mathbf{F}$ & Not well defined & & $293 \pm 99$ \\
\hline
\end{tabular}

${ }^{\mathrm{a}}$ TEM observations (Fig. 3)

${ }^{\mathrm{b}} 100 \mathrm{mM}$ HEPES-NaOH (pH 7.4) containing DMSO (<4.0 vol\%)
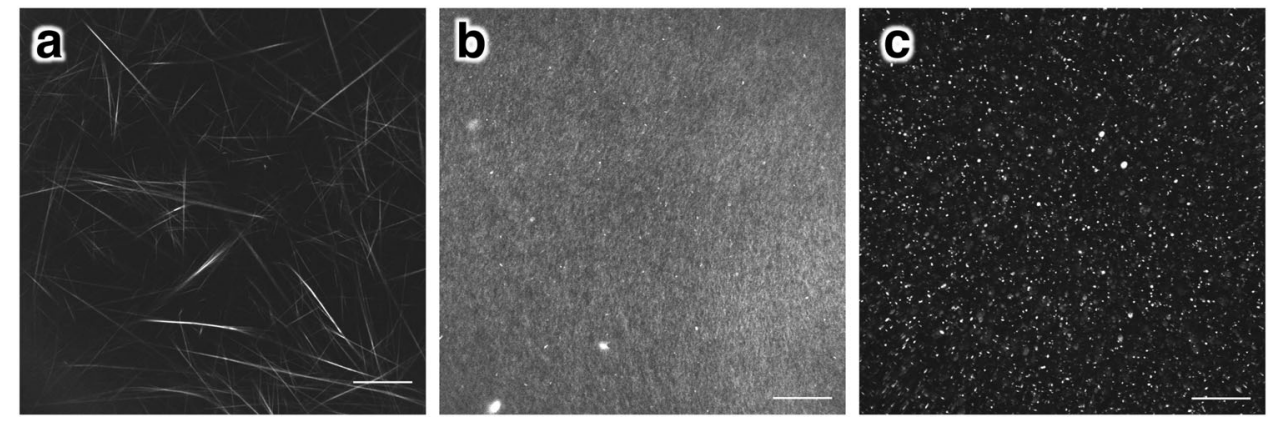
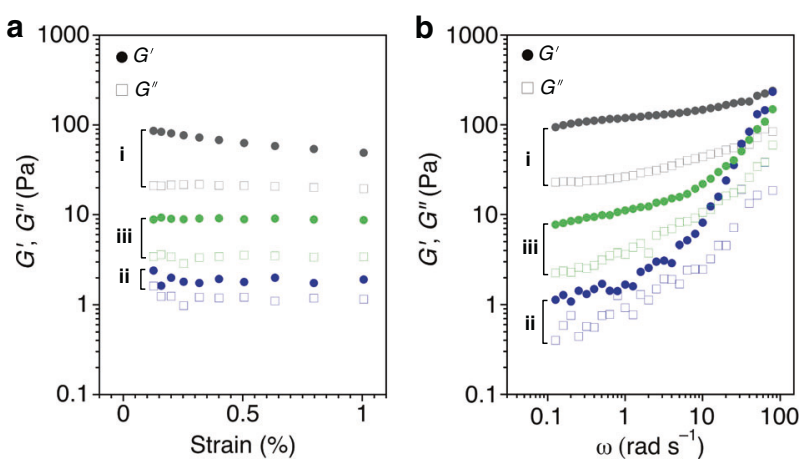

Fig. 6 Viscoelastic properties of hydrogels. (a) Strain sweep and (b) frequency sweep $(1.0 \mathrm{rad} / \mathrm{s}$ for strain sweep and $0.2 \%$ stain for frequency sweep) rheological properties of (i) Fmoc-FF, (ii) Fmoc-mFF, and (iii) Fmoc- ${ }^{\mathbf{D}} \mathbf{F m F}$ hydrogels $\left(G^{\prime}\right.$, storage modulus; $G^{\prime \prime}$, loss modulus). Conditions: [Fmoc-XXs] $=0.4 \mathrm{wt} \%, 100 \mathrm{mM}$ HEPES$\mathrm{NaOH}(\mathrm{pH} 7.4)$ containing DMSO (4.0 vol\%), $25^{\circ} \mathrm{C}$

of hydrogel formation, CLSM observations of the hydrogels of Fmoc-FF, Fmoc-mFF, and Fmoc- ${ }^{\mathbf{D}} \mathbf{F m F}$ were carried out. To stain the self-assembled structures, Nile red was added to the hydrogels [17]. As shown in Fig. 5a, Fmoc-FF showed networks composed of clearly discernible fibrous structures. On the other hand, Fmoc-mFF showed less clearly discernible fibrous structures (most likely owing to less bundled fibrous structures), as shown in Fig. 5b. These fiber network morphologies are consistent with those observed under TEM. In contrast, bright spots and their clusters were found almost uniformly for $\mathbf{F m o c}-{ }^{\mathbf{D}} \mathbf{F m F}$
(Fig. 5c), suggesting that clustered spherical nanostructures might be responsible for the hydrogel formation, as suggested by TEM.

The viscoelastic properties of Fmoc-FF, Fmoc-mFF, and

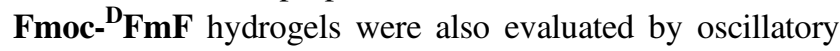
rheology measurements. As shown in Fig. 6a, the strainsweep oscillatory rheology of the three hydrogels displays a linear viscoelastic region. Figure $6 \mathrm{~b}$ shows the frequencysweep oscillatory rheology in the linear viscoelastic region. The storage modulus $\left(G^{\prime}\right)$ of Fmoc-FF (Fig. 6b-i) was significantly larger than the loss modulus $\left(G^{\prime \prime}\right)$, which is almost consistent with those reported previously $[12,13]$. In contrast, Fmoc-mFF (Fig. 6b-ii) and Fmoc- ${ }^{\mathbf{D}} \mathbf{F m F}$ (Fig. 6b-iii) showed smaller $G^{\prime}$ and $G^{\prime \prime}$ values than with Fmoc-FF. Nevertheless, the $G^{\prime}$ values were larger than the $G^{\prime \prime}$ values, indicating the formation of weak physical hydrogels. On the other hand, Fmoc-mFF and Fmoc- ${ }^{\mathbf{D}} \mathbf{F m F}$ hydrogels displayed relatively strong frequency dependences compared with the Fmoc-FF hydrogel (Fig. 6b). This difference could be attributed to the difference in their network structures (e.g., permanency), as suggested by TEM and CLSM observations. Theory for semiflexible polymers [40] predicts that the rigidity of a cross-linked gel is dependent on both the mesh size and the fibril bending modulus. According to the TEM and CLSM images, the fibril bending modulus of Fmoc-mFF appears to be smaller than that of Fmoc-FF, which might be consistent with the smaller $G^{\prime}$ of FmocmFF. On the other hand, Fmoc- ${ }^{\mathbf{D}} \mathbf{F m F}$ exhibited apparently different morphologies of supramolecular nanostructures, 
that is, clustered spherical nanostructures. Thus, the theoretical background to ascribe the rheological property should be applied after specialized experiments, which is beyond the scope of this study.

The self-assembling mode at the molecular level remains to be understood. According to the crystal structure of Fmoc-FF reported previously by Adams and coworkers [13], the $\pi$-stacking interactions of Fmoc groups and side chain phenyl groups, as well as the hydrogen bonding interactions among carbamate groups and amide groups, are crucial for giving rise to the one-dimensional self-assembled structure, reasonably corresponding to nanofiber formation. Preliminary molecular mechanics calculations (Fig. S11, electronic supplementary materials) suggest that the newly introduced methyl group of Fmoc-mFF at the $\mathrm{N}$-terminus might be accommodated in a one-dimensional self-assembled structure that is somewhat expanded compared to that of Fmoc-FF, whereas introducing the methyl group at the C-terminus in Fmoc-FmF could disturb the one-dimensional self-assembled structure by weakening the $\pi$-stacking interactions as well as the hydrogen bonding interactions. Further experimental and more detailed molecular modeling studies are required to elucidate the differences from the viewpoint of self-assembling structures at the molecular level.

\section{Conclusion}

In conclusion, this study revealed that the introduction of one or two methyl groups onto the $\alpha$ carbons of an FmocFF scaffold has a vital influence on the morphology of the supramolecular architecture at the nanometer scale as well as on the macroscopic properties (hydrogel or dispersion). Further research to elucidate the self-assembling modes of peptides comprising $\alpha$-methyl-amino acids leading to distinctive supramolecular nanostructures with biofunctional properties is in progress in our group.

\begin{abstract}
Acknowledgements MI sincerely thanks Prof. Keiji Maruoka (Kyoto University) and NAGASE \& CO. LTD (Japan) for kind donation of Fmoc-mF and $\mathbf{m F}$. We thank Mr. Koichiro M. Hirosawa and Prof. Kenichi G. N. Suzuki for their kind assistance in using CLSM. We acknowledge the Life Science Research Center and Research Equipment Sharing Promotion Center, Gifu University, for maintenance of the instruments and kind support. The authors would like to thank Enago (www.enago.jp) for the English language review.
\end{abstract}

\section{Compliance with ethical standards}

Conflict of interest The authors declare that they have no conflict of interest.

Publisher's note Springer Nature remains neutral with regard to jurisdictional claims in published maps and institutional affiliations.
Open Access This article is licensed under a Creative Commons Attribution 4.0 International License, which permits use, sharing, adaptation, distribution and reproduction in any medium or format, as long as you give appropriate credit to the original author(s) and the source, provide a link to the Creative Commons license, and indicate if changes were made. The images or other third party material in this article are included in the article's Creative Commons license, unless indicated otherwise in a credit line to the material. If material is not included in the article's Creative Commons license and your intended use is not permitted by statutory regulation or exceeds the permitted use, you will need to obtain permission directly from the copyright holder. To view a copy of this license, visit http://creativecommons. org/licenses/by/4.0/.

\section{References}

1. Zhang S. Fabrication of novel biomaterials through molecular self-assembly. Nat Biotech. 2003;21:1171-8.

2. Zhou J, Li J, Du X, Xu B. Supramolecular biofunctional materials. Biomaterials. 2017;129:1-27.

3. Makama P, Gazit E. Minimalistic peptide supramolecular coassembly: expanding the conformational space for nanotechnology. Chem Soc Rev. 2018;47:3406-20.

4. Fleming S, Ulijn RV. Design of nanostructures based on aromatic peptide amphiphiles. Chem Soc Rev. 2014;43:8150-77.

5. Ikeda M. Stimuli-responsive supramolecular systems guided by chemical reactions. Polym J. 2019;51:371-80.

6. Kimura S. Primary to quaternary structures of molecular assemblies. Polym J. 2019;51:739-51.

7. Tsuchiya K, Kurokawa N, Gimenez-Dejoz J, Gudeangadi PG, Masunaga H, Numata K. Periodic introduction of aromatic units in polypeptides via chemoenzymatic polymerization to yield specific secondary structures with high thermal stability. Polym J. 2019; 51:1287-98.

8. Mizuno Y, Furuya H. Volume shrinkage of polypeptide hybrid xerogels induced by a helix-sense inversion. Polym J. 2019;51: 337-44.

9. Tabata Y, Mitani S, Uji H, Imai T, Kimura S. The effect of macrodipole orientation on the piezoelectric response of cyclic $\beta$ peptide nanotube bundles on gold substrates. Polym J. 2019;51: 601-9.

10. Zhang Y, Gu H, Yang Z, Xu B. Supramolecular hydrogels respond to ligand-receptor interaction. J Am Chem Soc. 2003;125: 13680-1.

11. Jayawarna V, Ali M, Jowitt TA, Miller AF, Saiani A, Gough JE et al. Nanostructured hydrogels for three-dimensional cell culture through self-assembly of fluorenylmethoxycarbonyl-dipeptides. Adv Mater. 2006;18:611-4.

12. Mahler A, Reches M, Rechter M, Cohen S, Gazit E. Rigid, selfassembled hydrogel composed of a modified aromatic dipeptide. Adv Mater. 2006;18:1365-70.

13. Raeburn J, Mendoza-Cuenca C, Cattoz BN, Little MA, Terry AE, Cardoso AZ et al. The effect of solvent choice on the gelation and final hydrogel properties of Fmoc-diphenylalanine. Soft Mat. 2015;11:927-35.

14. Martin AD, Wojciechowski JP, Warren H, in het Panhuisb M, Thordarson P. Effect of heterocyclic capping groups on the selfassembly of a dipeptide hydrogel. Soft Mat. 2016;12:2700-7.

15. Ikeda M, Tanida T, Yoshii T, Hamachi I. Rational molecular design of stimulus-responsive supramolecular hydrogel based on dipeptides. Adv Mater. 2011;23:2819-22.

16. Ikeda M, Tanida T, Yoshii T, Kurotani K, Onogi S, Urayama K et al. Installing logic-gate response to a variety of biological substances in supramolecular hydrogel-enzyme hybrids. Nat Chem. 2014;6:511-8. 
17. Tsuzuki T, Kabumoto M, Arakawa H, Ikeda M. The effect of carbohydrate structures on the hydrogelation ability and morphology of self-assembled structures of peptide-carbohydrate conjugates in water. Org Biomol Chem. 2017;15:4595-600.

18. Görbitz $\mathrm{CH}$. Nanotube formation by hydrophobic dipeptides. Chem Eur J. 2001;7:5153-9.

19. Reches M, Gazit E. Casting metal nanowires within discrete selfassembled peptide nanotubes. Science. 2003;300:625-7.

20. Toniolo C, Bonora GM, Bavoso A, Benedetti E, di Blasio B, Pavone $\mathrm{V}$ et al. Preferred conformations of peptides containing $\alpha$, $\alpha$-disubstituted $\alpha$-amino acids. Biopolymers. 1983;22:205-15.

21. Tanaka M. Design and synthesis of chiral $\alpha, \alpha$-disubstituted amino acids and conformational study of their oligopeptides. Chem Pharm Bull. 2007;55:349-58.

22. Inai Y, Ousaka N, Okabe T. Mechanism for the noncovalent chiral domino effect: new paradigm for the chiral role of the n-terminal segment in a $33_{10}$-helix. J Am Chem Soc. 2003;125:8151-62.

23. Etienne MA, Aucoin JP, Fu Y, McCarley RL, Hammer RP. Stoichiometric inhibition of amyloid-protein aggregation with peptides containing alternating $\alpha, \alpha$-disubstituted amino acids. J Am Chem Soc. 2006;128:3522-3.

24. Mba M, Mazzier D, Silvestrini S, Toniolo C, Fatás P, Jiménez AI et al. Photocontrolled self-assembly of a bis-azobenzene-containing $\alpha$-amino acid. Chem Eur J. 2013;19:15841-6.

25. McDougall L, Draper ER, Beadle JD, Shipman M, Raubo P, Jamieson AG et al. Enzymatically-stable oxetane-based dipeptide hydrogels. Chem Commun. 2018;54:1793-6.

26. Mondal S, Adler-Abramovich L, Lampel A, Bram Y, Lipstman S, Gazit E. Formation of functional super-helical assemblies by constrained single heptad repeat. Nat Commun. 2015;6:8615.

27. Kitamura M, Shirakawa S, Maruoka K. Powerful chiral phasetransfer catalysts for the asymmetric synthesis of $\alpha$-alkyl- and $\alpha, \alpha$ dialkyl- $\alpha$-amino acids. Angew Chem Int Ed. 2005;44:1549-51.

28. Maruoka K. Practical aspects of recent asymmetric phase-transfer catalysis. Org Process Res Devel. 2008;12:679-87.

29. Di Valentin M, Albertini M, Dal Farra MG, Zurlo E, Orian L, Polimeno A et al. Light-induced porphyrin-based spectroscopic ruler for nanometer distance measurements. Chem Eur J. 2016; 22:17204-14.

30. Weiss RG, Terech P. Molecular gels: materials with self-assembled fibrillar networks. Dordrecht, The Netherlands: Springer; 2006. Ch 8.

31. Schindelin J, Arganda-Carreras I, Frise E, Kaynig V, Longair M, Pietzsch T et al. Fiji: an open-source platform for biological-image analysis. Nat Methods. 2012;9:676-82.

32. Rueden CT, Schindelin J, Hiner MC, DeZonia BE, Walter AE, Arena ET et al. ImageJ2: ImageJ for the next generation of scientific image data. BMC Bioinform. 2017;18:529.

33. Biancalana M, Koide S. Molecular mechanism of Thioflavin-T binding to amyloid fibrils. Biochim Biophys Acta Proteins Proteomics. 2010;1804:1405-12.

34. Garcia AM, Iglesias D, Parisi E, Styan KE, Waddington LJ, Deganutti C et al. Chirality effects on peptide self-assembly unraveled from molecules to materials. Chem. 2018;4:1862-76.

35. Marchesan S, Easton CD, Kushkaki F, Waddingtona L, Hartley PG. Tripeptide self-assembled hydrogels: unexpected twists of chirality. Chem Commun. 2012;48:2195-7.

36. Basu K, Baral A, Basak S, Dehsorkhi A, Nanda J, Bhunia D et al. Peptide based hydrogels for cancer drug release: modulation of stiffness, drug release and proteolytic stability of hydrogels by incorporating D-amino acid residue(s). Chem Commun. 2016; 52:5045-8.

37. Poon WCK. The physics of a model colloid-polymer mixture. J Phys Condens Matter. 2002;14:R859-80.

38. Ruan L, Zhang H, Luo H, Liu J, Tang F, Shi YK et al. Designed amphiphilic peptide forms stable nanoweb, slowly releases encapsulated hydrophobic drug, and accelerates animal hemostasis. Proc Natl Acad Sci USA. 2009;106:5105-10.

39. Le DHT, Hanamura R, Pham DH, Kato M, Tirrell DA, Okubo T et al. Self-assembly of elastin-mimetic double hydrophobic polypeptides. Biomacromolecules. 2013;14:1028-34.

40. Mackintosh FC, Kas J, Janmey PA. Elasticity of semiflexible biopolymer networks. Phys Rev Lett. 1995;75:4425-8. 